\title{
Over-representation of two specific haplotypes among chromosomes harbouring BRCA1 mutations
}

\author{
Ana Osorio*,1, Miguel de la Hoya ${ }^{2}$, Raquel Rodríguez-López ${ }^{1}$, Juan José Granizo ${ }^{3}$, \\ Orland Díez ${ }^{4}$, Ana Vega ${ }^{5}$, Mercedes Durán ${ }^{6}$, Angel Carracedo ${ }^{5}$, Montserrat Baiget ${ }^{4}$, \\ Trinidad Caldés ${ }^{2}$ and Javier Benítez ${ }^{1}$
}

\begin{abstract}
${ }^{1}$ Department of Human Genetics, Spanish National Cancer Center, Madrid, Spain; ${ }^{2}$ Molecular Oncology Laboratory, San Carlos University Hospital, Madrid, Spain; ${ }^{3}$ Medical Epidemiology Department, Jiménez Díaz Foundation, Madrid, Spain; ${ }^{4}$ Genetics Service, Sant Pau Hospital, Barcelona, Spain; ${ }^{5}$ Department of Molecular Medicine, Conxo Hospital, Santiago de Compostela, Spain; ${ }^{6}$ Department of Chemistry and Molecular Biology, Faculty of Medicine, Valladolid, Spain
\end{abstract}

The BRCA1 gene is included in a $200-400 \mathrm{~kb}$ region that is subjected to a recombination suppression mechanism; this region shows nearly complete linkage disequilibrium for a series of common biallelic polymorphisms, all of them with rarer allele frequency close to 0.4. These series of SNPs define two major haplotypes designated as class I and class II. In the present study, we have determined haplotype classes in the index case of 106 breast/ovarian cancer families previously screened for mutations in the BRCA genes and we have found that haplotype II (the less frequent in the control population) is over-represented among chromosomes harbouring mutations in BRCA1. In addition, we have defined a subtype of chromosomes characterized by haplotype I and one specific allele for the microsatellite marker D17S855, which are also more frequently associated with BRCA1 mutations. These findings may have important consequences for the selection of families with higher probabilities of carrying mutations in the BRCA1 gene.

European Journal of Human Genetics (2003) 11, 489-492. doi:10.1038/sj.ejhg.5200969

Keywords: BRCA1 mutation; haplotype; linkage disequilibrium; DNA repair; family selection; genetic testing

\section{Introduction}

Mutations in the BRCA genes are thought to account for about $30 \%$ of the breast and/or ovarian cancer families, ${ }^{1}$ and their alterations are scattered through the whole sequence of the genes (http://www.nhgri.nih.gov/Intramural_research/Lab_transfer/Bic/Member/index.html); this lack of hot-spots, in addition to the large size of the genes, makes its molecular analysis hard and expensive in most cases. For this reason, the identification of molecular markers that could help to select patients with high

*Correspondence: Dr Ana Osorio, Department of Human Genetics, Spanish National Cancer Center, C/Melchor Fernández Almagro 3, 28029 Madrid, Spain. Tel: +34 9122469 49; Fax: +34 9122469 23; E-mail: aosorio@cnio.es

Received 28 August 2002; revised 15 January 2003; accepted 15 January 2003 probabilities to carry BRCA mutations would be useful in making the screening more effective and faster. In this way, it has recently been observed in Spanish populations that BRCA1 mutations could be more frequently found in chromosomes that are defined by the presence of short alleles (139 and $141 \mathrm{bp}$ ) for the BRCA1 intragenic microsatellite marker D17S855. ${ }^{2}$

In the present study, we have determined two haplotypes associated with the BRCA1 gene in the index cases of 106 breast/ovarian cancer Spanish families by genotyping three BRCA1 SNPs included in a $200-400 \mathrm{~kb}$ region that is subjected to a recombination suppression mechanism. These SNPs define two major haplotypes, designated as class I and class II, that account for nearly all chromosomes in European and Asian populations. ${ }^{3}$ We have found that haplotype II is over-represented among chromosomes 
harbouring mutations in the BRCA1 gene $(\mathrm{OR}=2.8)$, while the shortest allele of the D17S855 microsatellite marker $(139 \mathrm{pb})$ is more frequently than expected associated with haplotype I $(\mathrm{OR}=3.5)$ among BRCA1 mutation carriers, defining a second haplotype over-represented among these chromosomes.

\section{Patient and methods Patients}

The analysis was performed in a serie of 106 high-risk breast and/or ovarian cancer families, selected using criteria previously described. ${ }^{4}$ The index case of each family had been previously screened for mutations in the BRCA1 and BRCA2 genes. ${ }^{4,5}$ Forty three families carried 22 different mutations in the BRCA1 gene (Table 1) and 63 did not carry mutations in either of the two genes. A total of 180 control individuals paired if sex and age with the cases, were used to establish the general frequencies of haplotypes I and II. The frequency of individual allele lengths for the D17S855 polymorphism in BRCA1 has been previously published. ${ }^{4}$

\section{Polymorphism genotyping}

Three BRCA1 biallelic polymorphisms were used to establish haplotype classes; the SNPs were IVS9-57T/ $\Delta \mathrm{T}, 4427 \mathrm{~T} /$ $\mathrm{C}$ and 5272+66G/A, located in intron 8, exon 13 and intron 18 , respectively. The three fragments were PCR amplified from genomic DNA using primers and PCR conditions previously described. ${ }^{4,5}$ When possible, one BRCA1 microsatellite marker, D17S855, was also genotyped. The PCR conditions and methods for fragment length analysis have been previously published. ${ }^{2}$

\section{Statistical analysis}

Comparison of allele frequencies was performed using $\chi^{2}$ test or Fisher exact test when necessary. The strength of the association between different categories was stated using OR and 95\% confidence interval calculated by the exact method. Epi Info v 6.0 and SPSS v 9.0 statistical packages were used for the analysis.

\section{Results}

We established haplotype classes I and II (defined by the three common alleles in the BRCA1 SNPs and the three rare alleles respectively) in 106 index cases from high-risk breast/ovarian cancer families (43 mutation carriers and 63 noncarriers), and 180 individuals from the general population. The genotypic frequencies found are summarized in Table 2. The frequencies for the three SNPs in the 180 individuals from the general population were the same as those described by Liu et al, ${ }^{3} P=0.66$ for the common allele and $q=0.34$ for the rare allele. The genotypic and allelic frequencies were very similar in this group $v s$ the 63 individuals not associated with mutations in BRCA1; so, we

Table 1 Haplotype assignment in the 21 distinct BRCA1 mutations in which the phase could be established

\begin{tabular}{|c|c|c|c|c|c|}
\hline BRCA1 mutation ${ }^{\mathrm{a}}$ & IVS-57delT & T4427C & $5272+66 G / A$ & $D 175855^{\mathrm{b}}$ & Class chromosome \\
\hline 185delAG & delT & C & $A$ & 0 & II \\
\hline 189insTGTC & delT & C & $A$ & -2 & II \\
\hline 234delCT & $\mathrm{T}$ & $\mathrm{T}$ & G & -2 & I \\
\hline $\mathrm{R} 71 \mathrm{G}$ & delT & C & A & +2 & II \\
\hline 243delA & $\mathrm{T}$ & $\mathrm{T}$ & G & -2 & I \\
\hline IVS6-1G/A & $\mathrm{T}$ & $\mathrm{T}$ & A & +6 & I \\
\hline 589delCT & $\mathrm{T}$ & $\mathrm{T}$ & G & +10 & I \\
\hline E445X & $\mathrm{T}$ & $\mathrm{T}$ & G & -2 & I \\
\hline 3022delTC & delT & C & $A$ & & II \\
\hline \multicolumn{6}{|l|}{ L974X $\mathrm{X}^{\mathrm{c}}$} \\
\hline 3598del11 & delT & C & $A$ & 0 & II \\
\hline 1135 insA & $\mathrm{T}$ & $\mathrm{T}$ & G & +2 & I \\
\hline 1241 delAC & delT & C & $A$ & 0 & II \\
\hline 1389insAG & delT & C & A & 0 & II \\
\hline 1693delA & $\mathrm{T}$ & $\mathrm{T}$ & G & $+4 /+8$ & I \\
\hline G1706E & delT & C & $A$ & 0 & II \\
\hline A1708E & $\mathrm{T}$ & $\mathrm{T}$ & G & -2 & I \\
\hline IVS18-1G/C & delT & $\mathrm{T}$ & G & +12 & II \\
\hline $5272+1 G / A$ & delT & C & $A$ & 0 & II \\
\hline IVS20+1G/A & $\mathrm{T}$ & $\mathrm{T}$ & G & +8 & I \\
\hline 5537delA & delT & C & $A$ & 0 & II \\
\hline E1836X & delT & C & A & 0 & II \\
\hline
\end{tabular}

a The mutations are ordered $5^{\prime}-3^{\prime}$ sense through the BRCA1 gene. All of them are designated by the nucleotide position, except the cases in which there is an amino-acid change, in which they are designated by the codon number.

${ }^{b}$ The allele lengths for the microsatellite markers D17S855 (GDB192761) have the same distribution as reported in Liu and Barker ${ }^{3}$ and are designated in the same way. The most common allele is designated as 0 (14 1).

'This was the only mutation in which the phase could not be established because there was only one carrier heterozygote I/II. 
Table 2 Genotype distribution in the 43 carriers of BRCA1 mutations vs the 63 noncarriers and the 180 individuals from the general population

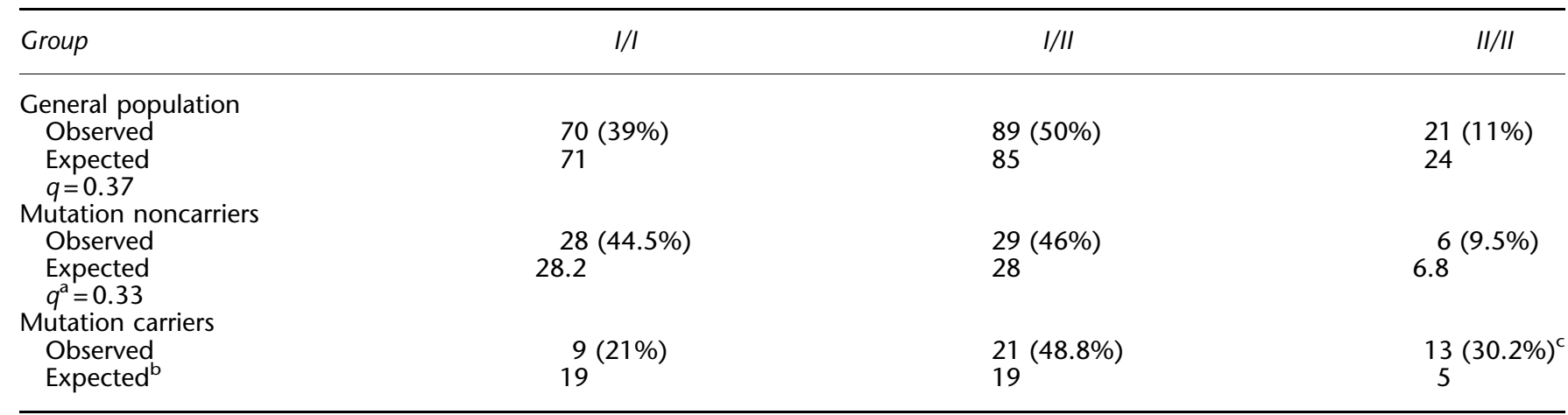

Haplotypes I and II consisted, respectively, of the 0.66 and 0.34 frequency allele at the three biallelic sites in the BRCA gene. ${ }^{3}$

${ }^{a}$ Allelic frequency of haplotype II in the group of noncarriers considered as controls for the study.

${ }^{\mathrm{b}}$ Genotypes expected considering the frequencies of the alleles in the control population.

${ }^{\mathrm{C}}$ This genotype was 4.12 times more frequent than in the control group $(\mathrm{Cl} 1.28-13.7, P=0.006)$.

considered these 63 individuals as the control group for all the comparisons performed in the study, because they represented the best matched group with the 43 mutation carriers (cases). In the 43 BRCA1 carriers, the homozygotes for haplotype II were clearly over-represented when compared with the 63 noncarriers (30.2\% vs 9.5\%). In terms of OR, homozygotes for haplotype II were 4.12 (CI $1.28-13.70, P=0.006)$ times more frequent in the group of cases than in the controls.

Among the 43 BRCA1 mutation carriers, only 22 different alterations were present. This could have biased the results, given that each recurrent mutation is usually found in its own specific haplotype. To avoid this problem, we tried to establish haplotype phase for the 22 distinct mutations found in our series and we found that the distinct BRCA1 mutations were more frequently associated with haplotype II than expected (57 vs 35\%), (OR 2.8, CI 0.99-7.84, $P=0.029$ ) (Table 1).

In 20 BRCA1 mutations we could complete haplotypes I and II with the D17S855 microsatellite marker (Table 1), whose shortest alleles (139 and $141 \mathrm{bp}$, designated as -2 and 0 , respectively) have recently been described to be associated with chromosomes harbouring BRCA1 mutations. $^{2}$ We confirmed that shortest alleles were concentrated in chromosomes carrying BRCA1 mutations (65 vs $40 \%, P<0.05)$; moreover, the distribution of allele -2 , the less frequently associated with haplotype I in the general population, ${ }^{3}$ was over-represented in the chromosomes with mutations associated with haplotype I (12 vs 44.4\%) (OR 3.5, CI 1.14-11.15, $P=0.049$ ).

\section{Discussion}

In the present study, we have established haplotypes I and II in the index cases of 106 breast/ovarian cancer families, by genotyping three of the SNPs located in the BRCA1 gene. Of these index cases, 43 carried mutations in the BRCA1 gene and 63 were negative for mutations either in BRCA1 or BRCA2 and were considered as controls for all the analyses performed. When analysing genotypic frequencies, the most significative finding was that the percentage of II/II (minor alleles) homozygotes among the BRCA1 carriers was about four times more frequent than in the controls $(30.2$ vs $9.5 \%)(\mathrm{OR}=4.12$, CI $1.28-$ $13.70, P=0.006$ ), while the I/I homozygotes (major alleles) were notably less frequent (21 vs 44\%). These results strongly suggest that the less common haplotype in the general population, haplotype II, was over-represented among the BRCA1 mutation carriers. Since these results could be biased by the presence of recurrent mutations linked to specific haplotypes, we established haplotype phases in 21 of 22 distinct mutations present in the 43 carriers, and found that the mutations were 2.8 times (CI $0.99-7.84, P=0.029)$ more frequently associated with haplotype II than expected, which was in agreement with our preliminary results.

On the other hand, it has been recently described that the shortest alleles (139 and $141 \mathrm{bp}$ ) of the D17S855 microsatellite (a highly informative BRCA1 intragenic marker) are more frequent among individuals carrying BRCA1 mutations. ${ }^{2}$ So, we genotyped this marker in our cases and found that the $139 \mathrm{bp}$ allele, designated as -2 , was 3.5 times more frequent than expected in mutations linked to haplotype I (CI $1.14-11.15, P=0.049$ ). So, these results define a second haplotype, which is also overrepresented among chromosomes harbouring BRCA1 mutations.

Altogether, these results suggest that the distinct BRCA1 mutations are not randomly distributed, but seem to be more frequently linked to two specific haplotypes that, 
interestingly, are less commonly found in the general population, haplotype II and haplotype I+(-2) allele. We do not have a clear explanation for this finding. If we were analysing one or a few mutations, we could think that simply these mutant alleles were older than the control, and haplotype II was more frequent in the time the mutations originated. However, the analysis and confirmation of our results in 21 different BRCA1 mutations argues against the possibility of a significant difference in the age of appearance between the mutant and the control alleles. That is why we tend to think that these specific haplotypes are linked to any factor that favours the presence of mutations.

A similar observation has recently been found for MLH1, one of the genes involved in DNA mismatch repair (MMR). Hutter et $\mathrm{l}^{6}$ hypothesize that although the polymorphisms constituting the haplotype are apparently neutral, the alleles could not be functionally equivalent and finally affect MMR efficiency and make mutations accumulate in the 'less-efficient' haplotype. Given that BRCA1 seems to be involved in DNA repair, ${ }^{7}$ the previous explanation could also be applicable to our case. However, most of these SNPs have been previously analysed in casecontrol studies to test its possible role as low penetrance variants and no association has been found. ${ }^{8}$ So, although we cannot discard their possible functional effects in mutation carriers, we think that the effect is not by the haplotype itself, but by some linked genetic factor, given the recombination suppression characteristic of the region.

Finally, our results are also interesting from a practical point of view, in order to select families with higher probabilities to be linked to this gene. In this way, given that the most commonly found mutations in Spanish populations are associated with haplotype II (data not shown), a patient from a high-risk family with a homozygous genotype for haplotype II would have a probability of $70 \%$ to be a carrier of mutations in the BRCA1 gene (Table 2).
In summary, our results for the BRCA1 gene represent the second report of mutations clustering in apparently neutral haplotypes. Although further studies increasing the number of mutations are necessary to confirm these findings in $\mathrm{MLH}^{6}{ }^{6}$ and BRCA1, it could be common to other tumour suppressor genes, especially those involved in DNA repair.

\section{Acknowledgements}

We thank Alicia Barroso for her excellent technical assistance. Ana Osorio is a fellow of Comunidad de Madrid and Raquel RodríguezLópez of Instituto de Salud Carlos III. This study was partially supported by FIS 01/0024 and SAF 01/0075.

\section{References}

1 Naathanson KL, Weber BL. Other breast cancer susceptibility genes: searching for more holy grail. Hum Mol Genet 2001; 10: 715-720.

2 de La Hoya M, Sulleiro S, Osorio A et al. Clustering of cancerrelated mutations in a subset of BRCA1 alleles: a study in the Spanish population. Int J Cancer 2002; 100: 618-619.

3 Liu X, Barker D. Evidence for effective suppression of recombination in the chromosome 17q21 segment spanning RNU2BRCA1. Am J Hum Genet 1999; 64: 1427-1439.

4 Osorio A, Barroso A, Martínez B et al. Molecular analysis of the BRCA1 and BRCA2 genes in 32 Spanish families with breast and or ovarian cancer. Br J Cancer 2000; 82: 1266-1270.

5 a de la Hoya M, Osorio A, Godin J et al. Association between BRCA1 and BRCA2 mutations and cancer phenotype in Spanish breast/ ovarian cancer families. Implications for genetic testing. Int $J$ Cancer 2002; 97: 466-471.

5b de la Hoya M, Peréz-Segura P, Van Orsow N et al. Spanish family study on hereditary breast and/or ovarian cancer. Analysis of the BRCA1 gene. Int J Cancer 2001; 91: 137-140.

6 Hutter P, Wijnen J, Rey-Berthod et al. An MLH1 haplotype is overrepresented on chromosomes carrying and HNPCC predisposing mutation in MLH1. J Med Genet 2002; 39: 323-327.

7 Venkitaraman AR. Cancer susceptibility and the functions of BRCA1 and BRCA2. Cell 2002; 108: 171-182.

8 Dunning AM, Chiano M, Smith NR. Common BRCA1 variants and susceptibility to breast and ovarian cancer in the general population. Hum Mol Genet 1997; 6: 285-289. 